\title{
A self-assembled peptide-appended naphthalene diimide: a fluorescent switch for sensing acid and base vapors
}

Article

Accepted Version

Gayen, K., Basu, K., Nandi, N., Sundar Das, K., HermidaMerino, D., Hamley, I. W. and Banerjee, A. (2019) A selfassembled peptide-appended naphthalene diimide: a fluorescent switch for sensing acid and base vapors.

ChemPlusChem, 84 (11). pp. 1673-1680. ISSN 2192-6506 doi: https://doi.org/10.1002/cplu.201900577 Available at https://centaur.reading.ac.uk/87454/

It is advisable to refer to the publisher's version if you intend to cite from the work. See Guidance on citing.

To link to this article DOI: http://dx.doi.org/10.1002/cplu.201900577

Publisher: Wiley

All outputs in CentAUR are protected by Intellectual Property Rights law, including copyright law. Copyright and IPR is retained by the creators or other copyright holders. Terms and conditions for use of this material are defined in the End User Agreement. 


\section{CentAUR}

Central Archive at the University of Reading

Reading's research outputs online 


\title{
A Self-Assembled Peptide-Appended Naphthalene Diimide: A Fluorescent Switch for Sensing Volatile Acid and Basic Vapors
}

\author{
Kousik Gayen, ${ }^{\text {[a] }}$ Kingshuk Basu, ${ }^{[a]}$ Nibedita Nandi, ${ }^{[a]}$ Krishna Sundar Das, ${ }^{[b]}$ Daniel Hermida- \\ Merino, ${ }^{[c]}$ lan W. Hamley ${ }^{[d]}$ and Arindam Banerjee ${ }^{*[a]}$
}

\begin{abstract}
A histidine containing peptide appended naphthalenediimide (NDI) based bola-amphiphilic molecule (NDIP) has been found to form fluorescent hydrogel in phosphate buffer and organogels with benzenoid solvents. These gels were characterized by several spectroscopic and microscopic technique like FT-IR, HRTEM, powder X-ray diffraction and small-angle X-ray scattering, UV$V$ is and fluorescence studies. The gelator molecule exhibits no significant fluorescence in the xerogel state, while it turns into a significant fluorescent (bright cyan) color in presence of volatile organic/inorganic acid vapors and this cyan color vanishes in presence of base (ammonia vapors). A paper strip based method is able to detect hazardous volatile acid and base vapors using this self-assembled fluorescent material that is economically viable, easily detectable through a naked eye and reusable several times.
\end{abstract}

\section{Introduction}

In the last few decades, $\pi$ - conjugated organic molecular functional aggregates have attracted a lot of attention to the scientists due to their interesting properties and wide spread applications in organic light emitting diodes ${ }^{[1]}$, field effect transistors ${ }^{[2]}$, solar cells ${ }^{[3]}$ and others ${ }^{[4]}$. Among the rylene dyes, naphthalenediimides (NDI) are special, owing to their redox active behavior, chemical robustness, molecular planarity, $\pi$ acidity and n-type semiconducting nature ${ }^{[5]}$ compared to other longer congeners (perylenes, terylenes, etc). These molecules are promoted to form aggregates and gels. These types of supramolecular aggregates find many potential applications in photovoltaic devices ${ }^{[6]}$, sensing ${ }^{[7]}$, bioprobes ${ }^{[8]}$ and others ${ }^{[9]}$. However, the low quantum yield in monomeric state of NDI based molecule limits their application. There are several ways to overcome this stumbling block including functionalization in

\footnotetext{
[a] K. Gayan, K. Basu, N. Nandi and Prof. A. Banerjee School of Biological Sciences, Indian Association for the Cultivation of Science, Jadavpur, Kolkata-7000032, India

E-mail: bcab@iacs.res.in

[b] K. S. Das

School of Chemical Sciences

Indian Association for the Cultivation of Science, Jadavpur, Kolkata7000032, India

[c] D. Hermida-Merino

ESRF - The European Synchrotron, 38043 Grenoble Cedex France.

[d] Prof. I. W. Hamley

Department of Chemistry, University of Reading, Whiteknights, Reading, RG6, 6AD, UK
}

Supporting information for this article is given via a link at the end of the document.((Please delete this text if not appropriate)) the core position ${ }^{[10]}$ and aggregation induced enhanced emission (AIEE) of NDI based molecules ${ }^{[11]}$ in a suitable solvent system. Peptide appended NDIs belong to a special category due to their self-assembling nature by various non-covalent interactions including $\pi-\pi$ interaction through NDI core, hydrogen bonding among the peptide backbones, van der Waals interaction of side chains and hydrophobic interactions to form soft functional materials with fascinating properties. There are several examples of the assembled peptide based soft functional materials ${ }^{[12]}$ and also a few studies have been directed to amino acid/ peptide conjugated NDI molecules ${ }^{[13]}$ that lead to the formation of fluorescent materials in a suitable solvent system.

Hazardous volatile acids are extensively used in research laboratories and chemical industries for synthesizing many useful compounds including various household items including cosmetics, fertilizers, detergents, and many others. These acids are highly corrosive for skin and eyes, and inhalation of these vapors damages the internal organs. Therefore, detection and removal of these acid vapors are absolutely necessary for keeping the environment safe and healthy. A number of attempts have been made to design and construct effective materials to detect of harmful acid vapors. There are several methods including colorimetric ${ }^{[14]}$, photometric ${ }^{[15]}$ and fluorometric $^{[16]}$ assays to detect hazardous acid vapors. There are a few examples of NDI based fluorescence "turn on/off" for detecting analytes including toxic metal ions ${ }^{[17]}$, explosives ${ }^{[18]}$ amine vapors ${ }^{[19]}$ and also biological motifs such as Gquadruplexes ${ }^{[20]}$.

However, to the best of our knowledge there is no report for sensing hazardous acid vapors with a fluorescence 'turn on' method by using naphthalenediimide derivatives. So, there is a genuine need for the design and construction of NDI based sensors to detect environmentally hazardous acid vapors efficiently in an economically viable manner.

In this study, a peptide-appended NDI-based molecule, NDIP has been synthesized, which forms a fluorescent hydrogel at $\mathrm{pH}$ 7.46 in phosphate buffer solution as well as in organic aromatic solvents like benzene, toluene or xylene. The fluorescence was quenched upon drying the corresponding gel solvents. Interestingly, these dried gels exhibit a wonderful application for detection of various volatile acid vapors $\left(\mathrm{HCl}, \mathrm{H}_{2} \mathrm{SO}_{4}\right.$, trifluoroacetic acid (TFA), $\mathrm{HCOOH}$, acetic acid). A paper strip impregnated with NDIP was used to detect various volatile acid vapors by 'turn on' fluorescence and ammonia vapor by 'turn off' fluorescence. This represents the first demonstration of a new peptide-appended NDI-based compound as a fluorescence switch for detecting vapors of acid and base with recyclability at least 7 times. 


\section{Results and Discussion}

A histidine-containing peptide-appended naphthalenediimidebased molecule, NDIP (Figure 1) has been synthesized, purified, characterized (Supporting information) and studied for gelation in aqueous as well as organic medium. Most of the NDI derivatives form aggregates in organic solvent due to strong $\pi-\pi$ interactions involving the naphthyl rings. Other interactions like hydrogen bonding among the peptide units, van der Waals interaction involving the side chain of the NDIP molecules can also play a role for gelation ${ }^{[9 b, 11 a-c]}$. In this study, the molecule NDIP is bola amphiphilic in nature due to the presence of two imidazole moieties of histidine residue at the termini and centrally located NDI core as well as oligomethylene

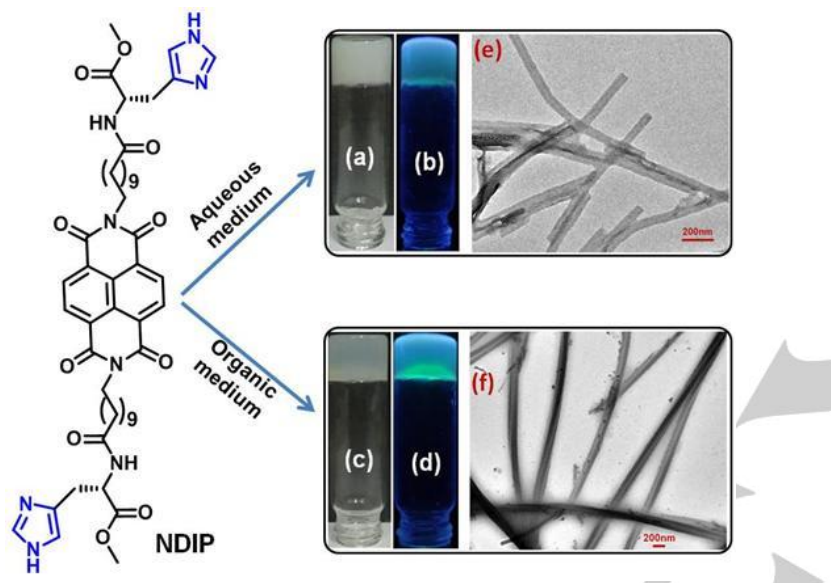

Figure 1. Chemical structure of NDIP. Vial picture of hydrogel formed by NDIP (a) at daylight and (b) under UV-light ( $\left.\lambda_{\max } 365 \mathrm{~nm}\right)$. Vial picture of toluene gel NDIP (c) at daylight and (d) under UV-light $\left(\lambda_{\max } 365 \mathrm{~nm}\right)$. (e) and (f) are the HR-TEM images of the xerogels of hydro and organogels respectively (scale bar $200 \mathrm{~nm}$ ).

units from central part of the molecule. So, this peptide appended NDI derivative form aggregates both in aqueous solution as well as in organic solvent to form gels under suitable condition.

\section{Morphological Study}

A high resolution transmission electron microscopic (HR-TEM) study of the organo and hydrogels reveals a nanotubular morphology with various wall thickness in both cases (Figure 1e, $1 f$ and Figure S4, S5). These nanotubes are entangled to each other to form a network structure that is responsible for gelation. These tubes are several micrometers in length and the inner diameters of these tubes obtained from the hydrogel and organogel are found to be within 15-20 nm and 30-45 nm respectively. However, the total diameter (inner + outer) of these tubes lies between $60-70 \mathrm{~nm}$ and $110-130 \mathrm{~nm}$ for hydrogel and organogel respectively. This variation regarding the thickness of the nanotube in two different media (aqueous as well as in organic medium) may be due to the distinct nature of packing pattern in their respective assembled/gel states.

\section{Fourier Transform Infrared (FT-IR) Analysis}

Fourier transform infrared (FT-IR) spectroscopic studies were carried out to obtain information on the hydrogen bonding interactions among the gelator molecules in the self-assembled state. The significant peaks were obtained at 3450, 3298, 1732, 1704, 1654, $1577 \mathrm{~cm}^{-1}$ for the hydrogel (in phosphate buffer solution $\mathrm{pH}$ 7.46) of NDIP in the FT-IR spectra (Figure S6).

The peak at $3450,3298 \mathrm{~cm}^{-1}$ can be assigned to non-hydrogen bonding and hydrogen bonding $\mathrm{N}-\mathrm{H}$ stretching ${ }^{[21]}$, while peaks at $1654,1577 \mathrm{~cm}^{-1}$ stretching are due to amide $\mathrm{C}=\mathrm{O}$ stretching and $\mathrm{N}-\mathrm{H}$ bending respectively. Similar peaks were obtained for the organogel in toluene at $3298,1732,1704,1654,1577 \mathrm{~cm}^{-1}$. But, in this case the peak position corresponding to non-hydrogen bonded $\mathrm{N}-\mathrm{H}$ stretching was absent. The peaks corresponding to $1240 \mathrm{~cm}^{-1}$ and $1342 \mathrm{~cm}^{-1}$ are due to the imide stretching frequencies of NDI unit. Moreover, FT-IR study was carried out in the monomeric state (non-aggregated state) of the NDIP in dimethyl sulfoxide medium (Figure S6b) to compare the hydrogen bonding interaction between monomeric state and aggregated state (hydrogel and organogel). In the Figure S6, the peak appears at $3455 \mathrm{~cm}^{-1}$ and this is due to the non-hydrogen bonded $\mathrm{N}-\mathrm{H}$ stretching of the peptide unit. From the FT-IR spectra analysis, it is apparent that in DMSO medium only nonhydrogen bonded $\mathrm{N}-\mathrm{H}$ stretching is present. However, both hydrogen-bonded and non-hydrogen bonded $\mathrm{N}-\mathrm{H}$ peaks are observed for the hydrogel and in organol-gel state all the $\mathrm{N}-\mathrm{Hs}$ are fully hydrogen bonded. There is no sign of non-hydrogen bonded peak in the organo-gel. So, it can be anticipated that hydrogen bonding interaction plays an important role during selfassociation and gelation of the gelator molecule, as it is evident from the respective IR spectra (Figure S6).

\section{NMR Experiment in the gel state}

To confirm non-covalent interactions (namely hydrogen bonding interaction) among the gelator molecules within the selfassembled state, temperature dependent ${ }^{1} \mathrm{H}$ NMR experiments were performed using deuterated benzene as a solvent. The peptide NDIP forms an organogel in the solvent system 5\% methanol- $d_{4}$ and $95 \%$ benzene- $d_{6}(v / v)$. So, temperature dependent ${ }^{1} \mathrm{H}$ NMR experiment was carried out in that solvent composition to examine the hydrogen bonding nature of peptide $\mathrm{NHs}$ in the self-assembled organogel state in that particular solvent mixture. The hydrogen bonding takes place among the amide $\mathrm{NH}$ and $\mathrm{C}=\mathrm{O}$ of the peptide backbone. With the increase in temperature the hydrogen bonding strength is weakening. This is exemplified by the steady and gradual up-field shift of amide $\mathrm{N}-\mathrm{H}$ protons with an increase in temperature from $25^{\circ} \mathrm{C}$ to $70^{\circ} \mathrm{C}$. From the Figure 2, it is seen that the $\delta$ value of chemical shift of the corresponding proton appeared at $6.62 \mathrm{ppm}$ at room temperature $\left(25^{\circ} \mathrm{C}\right)$. The $\delta$ value of this amide $\mathrm{NH}$ proton is gradually shifted to the up field upon increasing temperature and 
these $\delta$ values are at $6.54,6.50$ and $6.47 \mathrm{ppm}$ at the temperatures $40^{\circ} \mathrm{C}, 55^{\circ} \mathrm{C}$ and $70^{\circ} \mathrm{C}$ respectively. So, it can be stated that the temperature dependent ${ }^{1} \mathrm{H}$ NMR experiment is a powerful tool for understanding the involvement of hydrogen bonding interaction in the gel phase and it is observed that the gel melts and converts to sol at the $70^{\circ} \mathrm{C}$ temperature and the $\Delta \delta$ value (the difference in chemical shifts between first $\left(25^{\circ} \mathrm{C}\right.$ ) and last $\left(70^{\circ} \mathrm{C}\right)$ readings is $0.15 \mathrm{ppm}$ indicating the complete disruption of this hydrogen bonding with an increase in temperature and it is also occurred with the gel to sol transition.

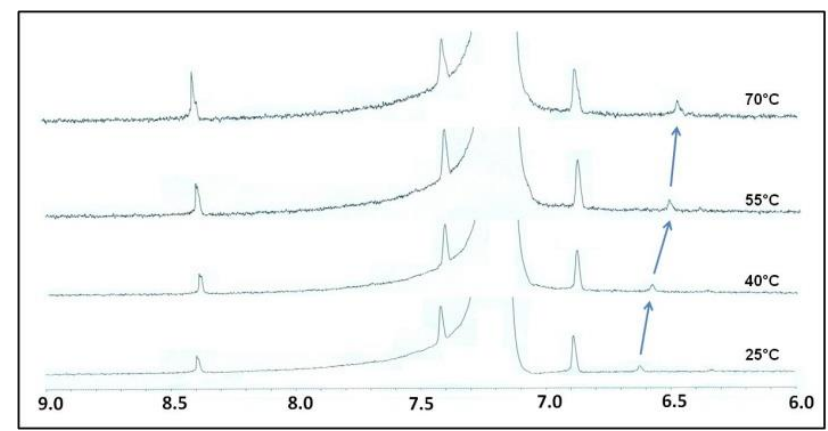

Figure 2. Temperature dependent ${ }^{1} \mathrm{H}$ NMR spectrum of NDIP in gel state in $\mathrm{C}_{6} \mathrm{D}_{6}$.

\section{Small-angle X-ray scattering (SAXS) Study}

Small angle X-ray scattering (SAXS) experiments were performed for NDIP in the hydrogel and organogels. As shown in Figure 1e and 1f, the gelator molecule forms nanotubular structures in both gels. For the hydrogel, the form factor fit is to a hollow cylinder (nanotube) form factor with core radius (16.3 \pm 1.2) $\mathrm{nm}$ and wall thickness $7.2 \mathrm{~nm}$, i.e. total tube diameter of 47 $\mathrm{nm}$. This is in good agreement with the TEM images. But for the organogel (in toluene) the form factor is fit using the same model with core radius $(13.7 \pm 1.8) \mathrm{nm}$ and wall thickness $12.7 \mathrm{~nm}$ (Figure S7). This leads to a tube diameter lower than that estimated by TEM. This is ascribed to drying effects in preparation of the TEM samples. We can conclude that both SAXS data as well as TEM images show nanotubular structures for NDIP in the aggregated state for both hydrogels and organo gels $^{[22]}$.

\section{Wide angle Powder X-ray Diffraction (WPXRD) Studies}

Wide-angle powder XRD studies (WPXRD) of the xerogels were performed to explore the packing arrangement of the molecules within the self-assembled network structure. The calculated molecular length (D) of NDIP is $42.77 \AA$ as obtained using ChemBioDraw 3D software. In the wide-angle region for the hydrogel (Figure 3a) the intense peaks appear at $2 \theta=17.02^{\circ}$ $(d=5.20 \AA), 2 \theta=18.50^{\circ}(d=4.79 \AA), 2 \theta=19.34^{\circ}(d=4.58 \AA)$ can be assigned to $D / 8, D / 9, D / 10$. Similarly for the organogel (Figure $3 b)$, periodic peaks at $2 \theta=11.77^{\circ}(d=7.51 \AA), 2 \theta=14.71^{\circ}(d=6.01$ $\AA), 2 \theta=16.21^{\circ}(d=5.46 \AA)$ can be assigned to $D / 6, D / 7, D / 8$.
These results indicate a lamellar type of arrangement for the molecules in the self-assembled network structure in both organo- and hydrogels. The peaks corresponding to $2 \theta=8.59^{\circ}$ $(d=10.28 \AA)$ and $2 \theta=18.43^{\circ}(d=4.81 \AA)$ are due to inter-sheet and inter-strand distance of a $\beta$-sheet-like structure of the aggregated molecule NDIP in organic medium ${ }^{[23]}$. However, only sheet-like assembly was noticed in aqueous medium, the peak at $2 \theta=18.50^{\circ}(d=4.79 \AA)$ is obtained from the assembled state from hydrogel. The $\pi-\pi$ distance between the imidazole moieties, naphthalene core of the molecule in the hydrogel and organogel have been found in the region at $2 \theta=22.62^{\circ} \quad(d=3.93 \AA)$, $2 \theta=23.46^{\circ}(d=3.79 \AA)$ and $2 \theta=21.16^{\circ}(d=4.19 \AA), 2 \theta=21.87^{\circ}$ $(d=4.06 \AA$ ) respectively (figure 3 ). From the above data it can be said that the arrangement among the gelator molecules is somewhat different in organogel than that in the hydrogel.

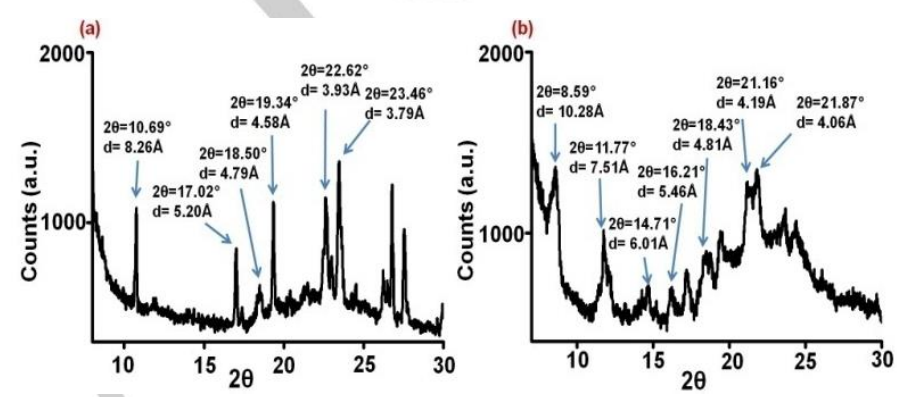

Figure 3. Wide angle powder XRD (WPXRD) spectra for (a) hydrogel (phosphate buffer solution $\mathrm{pH}$ 7.46) (b) organo gel (toluene).

A probable model for the nanotube formation in these two media has been constructed based on HR-TEM, SAXS, WPXRD studies (Figure 4).

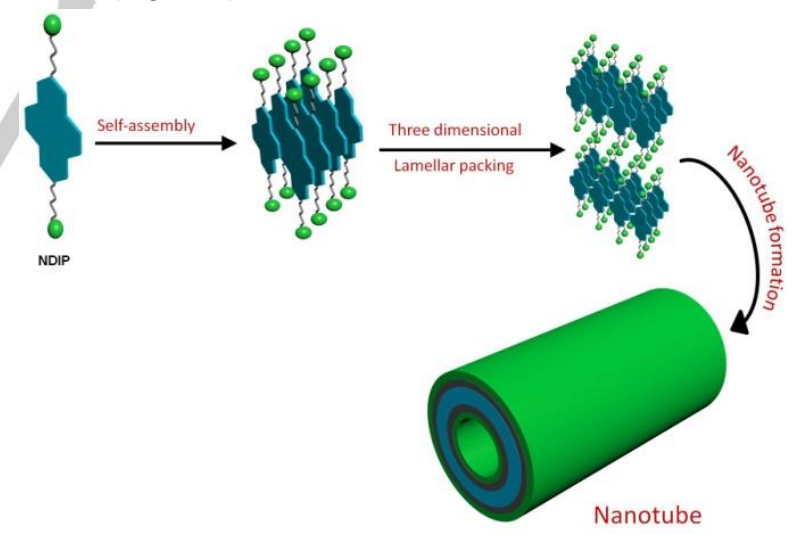

Figure 4. Probable model for the nanotube formation of NDIP in aqueous as well as in organic medium.

\section{Rheological Studies}

To obtain insight about the stiffness and viscoelastic property of the hydrogel and the organogel from NDIP, different rheological experiments were carried out at a fixed concentration $0.52 \%$ $(\mathrm{w} / \mathrm{v})$ and constant strain about $0.1 \%$ (Figure 5). The frequency sweep experiment was performed for organogel and hydrogel separately and the results are presented in the Figure 5. It was 
observed from the results that the storage modulus $\left(G^{\prime}\right)$ is always greater than the loss modulus (G") and both are only weakly dependent on frequency. Both these features are characteristics of gels. Interestingly, it has been noticed that the mechanical strength of the organogel is higher than that of hydrogel at a fixed angular frequency. It is observed from the FT-IR studies that both non-hydrogen and hydrogen bonded $\mathrm{N}$ $\mathrm{H}$ stretching frequencies are present in the case of hydrogel, while the organogel shows only $\mathrm{N}-\mathrm{H}$ stretching frequency corresponding to hydrogen bonded $\mathrm{N}-\mathrm{H}$ peaks. Thus, in the organogel all $\mathrm{N}-\mathrm{Hs}$ of the gelator molecule are hydrogen bonded, whereas all $\mathrm{N}-\mathrm{Hs}$ do not participate in $\mathrm{H}$ - bonding in the hydrogel state. This may be the reason for the observed greater gel stiffness for the organogel. To find the linear viscoelastic region (LVR), we carried out amplitude sweep experiments for both gels (Figure S8). For the hydrogel gel the LVR resides between $0.01 \%$ to $0.75 \%$ shear strain and the concerned region has been highlighted and $\mathrm{G}^{\prime}$ (storage modulus), G" (loss modulus) crossover takes place upon application of $\sim 8 \%$ shear strain. For the organogel (in toluene) the LVR resides between $0.01 \%$ to $0.63 \%$ shear strain and the LVR has been highlighted and $G^{\prime}$ (storage modulus), G" (loss modulus) crossover takes place upon application of $\sim 30 \%$ shear strain.

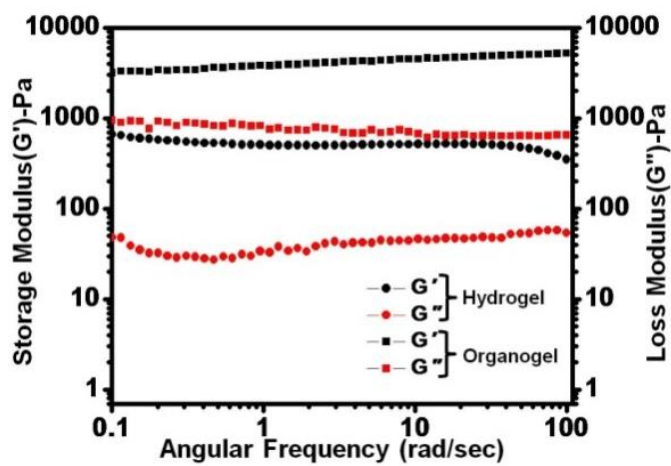

Figure 5. Frequency sweep rheology data for hydrogel and organogel at a constant strain about $0.1 \%$.

\section{UV-Vis Study}

UV-Vis spectra were measured to examine the change of selfassembly pattern of the chromophoric moiety of NDIP from the monomeric to an aggregated state at a fixed concentration $(0.05$ $\mathrm{mM})$. The molecule NDIP shows monomeric nature in hexafluoroisopropanol (HFIP), a well known highly polar solvent. In the UV-vis spectrum (Figure 6a) sharp absorption peaks were observed at $361 \mathrm{~nm}$ and $381 \mathrm{~nm}$ with a shoulder at around 341 $\mathrm{nm}$. This is due to $\pi-\pi^{*}$ transition along the long polarized axis of the NDI chromophore in hexafluoroisopropanol (HFIP). The selfassembly of NDIP was examined by the gradual addition of toluene or aqueous buffer solution to the monomeric solution of HFIP at a fixed concentration of the gelator molecule. It is found that upon the addition of aqueous buffer into the solution of NDIP in HFIP the higher wavelength peak starts to shift at a composition 10:90 (HFIP: phosphate buffer) by $5 \mathrm{~nm}$ and at a composition of 2:98 (HFIP: phosphate buffer) the peak is red shifted by $10 \mathrm{~nm}$ with decrease in intensity (Figure 6a). This clearly suggests the formation of J-aggregated species in the aqueous buffer solution. A similar result is observed upon adding toluene into the monomeric solution of NDIP in HFIP (figure $6 \mathrm{c}$ ). It has been found that at a composition of 2:98 (HFIP: toluene) the peak which appeared at $382 \mathrm{~nm}$ is $10 \mathrm{~nm}$ red shifted with a diminishing intensity. This clearly indicates the presence of $\mathrm{J}$ - aggregates in toluene.

\section{Fluorescence study}

In the monomeric state the gelator molecule NDIP shows a very feeble violet fluorescence in HFIP, while it exhibits a bright blue fluorescence in the hydrogel and also in the organogel state under illumination of UV light $\left(\lambda_{\max }\right.$ at $\left.365 \mathrm{~nm}\right)$. This prompted a more detailed fluorescence study of NDIP in the nonaggregating as well as in the aggregating solvent (aqueous phosphate buffer and toluene separately). A significant change of fluorescence intensity (enhancement) was noticed upon gradual addition of aggregating solvent like buffer solution $(\mathrm{pH}$ 7.46) or aromatic solvent (toluene) to the HFIP. The enhancement of fluorescent intensity in the aggregated state is due to the intrinsic phenomenon, aggregation induced enhance emission (AIEE) of the molecule NDIP. The correlation of fluorescence property and aggregation behavior of the compound was investigated in solvent mixture (HFIP and phosphate buffer/aromatic solvent, toluene). From Figure $6 \mathrm{~d}$, it is evident that significant fluorescence is observed at the solvent composition 20:80 (HFIP: toluene) and the highest fluorescence intensity was found when the ratio of (HFIP: toluene) was 2:98. A sharp emission peak appeared at $423 \mathrm{~nm}$ for the toluene gel. A similar fluorescence spectral feature of the aggregating peptide NDIP was observed in phosphate buffer medium (hydrogel state, Figure 6c). Interestingly, the hydrogel emits blue light with an emission maximum $433 \mathrm{~nm}$ upon excitation at 365 $\mathrm{nm}$. The photoluminoscence study thus clearly suggests that the fluorescence can originate due to the aggregation of NDIP in aqueous medium as well as in toluene. Another noticeable feature is that the compound NDIP in solid state or in the xerogel state does not exhibit any significant fluorescence. 

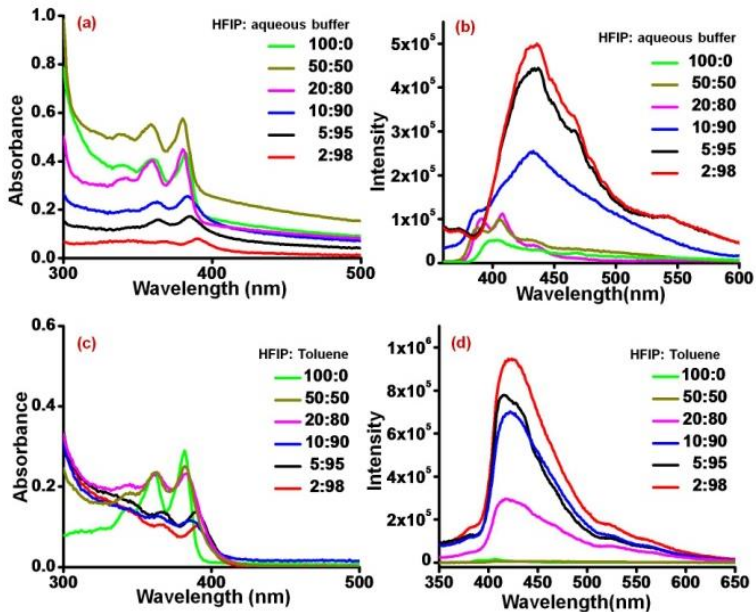

Figure 6. (a) UV-Vis absorption and (b) fluorescent emission spectra of NDIP in different compositions of the monomeric solvent hexafluoroisopropanol (HFIP) and phosphate buffer solution pH 7.46. (c) UV-vis absorption and (d) fluorescent emission spectra of NDIP in different compositions of the monomeric solvent hexafluoroisopropanol (HFIP) and toluene.

\section{Time-correlated Single Photon Counting (TCSPC) study}

A time-correlated single photon counting experiment was carried out for the measurement of fluorescence life time and decay nature of the gelator molecule NDIP in the monomeric state and aggregated state (Figure S9). The excitation and emission monochromator were set at $340 \mathrm{~nm}$ and $420-460 \mathrm{~nm}$ respectively for all the cases (monomeric and aggregated states). In the monomeric state of the molecule NDIP a short lived decay nature with an average life time 0.0782 ns was noticed. For NDIP in buffers solution a bi-exponential decay was observed with a substantially longer average lifetime of $1.17 \mathrm{~ns}$ (Figure S9) compared to its monomeric average lifetime. Similarly, in case of NDIP in toluene, the resultant decay profiles also showed a bi-exponential decay with a substantially longer average lifetime of $1.01 \mathrm{~ns}$ (Figure S9) compared to its monomer state lifetime. Therefore, it is evident that the excited state complexes (in buffer and toluene) of NDIP are significantly more stable than that of their monomeric state.

\section{Sensing Experiment}

In this study, the fluorescence behavior of the compound NDIP in response to different volatile organic/inorganic acid vapor has been investigated thoroughly. The gelator molecule NDIP contains two imidazole rings. So, it can be envisaged that the nitrogen lone pair of imidazole ring can interact with protic acids and this can affect the self-assembly of NDIP or photophysical process (PET, TICT) of the molecule that leads to fluorescence property. The hydrogel and organogel individually shows a bright blue fluorescence. However, in the solid state or xerogel state (dried gel) almost no fluorescence is observed.
We further explored whether the compound NDIP shows any acid-base responsive fluorescence behavior or not. A thin film was prepared by drop casting of the organogel on a glass plate and this exhibits very weak fluorescence centered at $440 \mathrm{~nm}$ upon UV irradiation $\left(\lambda_{\max }=365 \mathrm{~nm}\right.$ ) (figure $7 \mathrm{~b}$ ). Several volatile organic and inorganic acids including formic acid, acetic acid, trifluoreacetic acid, $\mathrm{HCl}, \mathrm{HNO}_{3}$, and $\mathrm{H}_{2} \mathrm{SO}_{4}$ have been used for this sensing. Aliquots of $100 \mu \mathrm{L}$ of the above mention acids were placed in the bottom of a thin layer chromatography (TLC) chamber and then a thin film of NDIP on a glass plate was placed at the top of TLC chamber for $5 \mathrm{~min}$. Interestingly, it was found that non fluorescent thin film becomes strong cyan fluorescent in the presence of acid vapors upon irradiation with a UV lamp at $365 \mathrm{~nm}$ (Figure 7b). Spectroscopic observation shows that appearance of a peak at $500 \mathrm{~nm}$ upon excitation at $365 \mathrm{~nm}$ (Figure 7a).

Based on the glass plate assay of the acid vapor detection, a fruitful method of paper based environmental sensor has been developed to detect both acid and base vapor by fluorescence turn "on" and "off". A paper strip based sensor simply acts as a low cost device and it is easily visible by naked eye. A simple Whatman 1 filter paper with dimension $5 \mathrm{~cm} \times 0.5 \mathrm{~cm}$ was used. The filter paper was coated by the compound (dissolved in $10 \%$ methanol and chloroform) from the $1 \mathrm{~cm}$ bottom part and it was dried in an air oven for 10 minutes. No fluorescence was found (Figure 7c) when the compound coated filter paper was held under UV light (illuminated at $365 \mathrm{~nm}$ ).
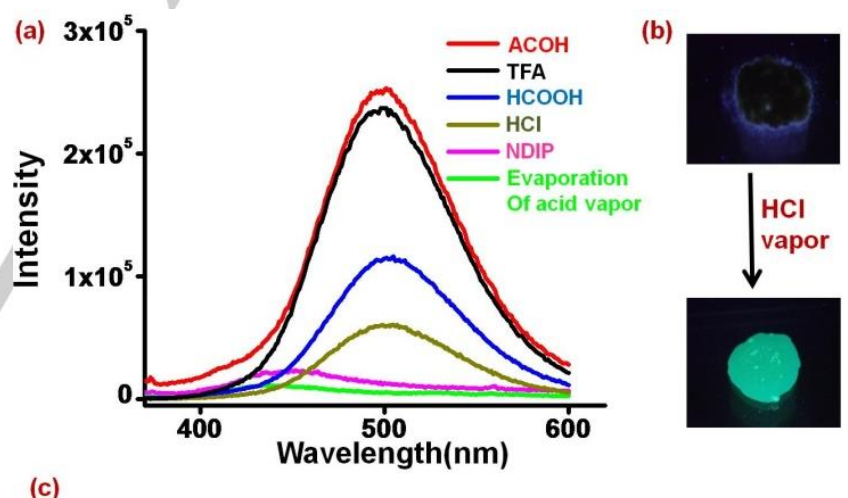

(c)

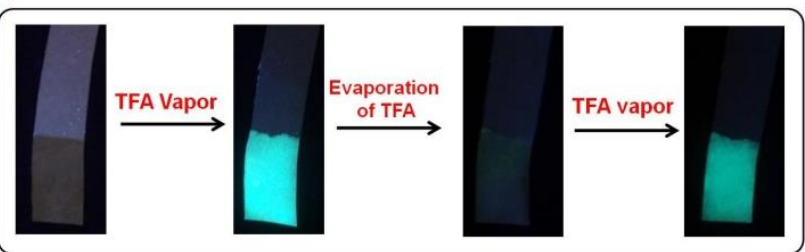

Figure 7. (a) Fluorescence spectra of NDIP xerogels upon exposure to different kinds of volatile acid vapors. (b) Fluorescence 'turn on' of NDIP xerogels upon exposure to $\mathrm{HCl}$ vapor. (c) Fluorescence on/off behavior by exposure and evaporation of acid vapor using a paper strip impregnated with NDIP 
Then the filter paper was exposed to any of the above mentioned acid vapors and checked in the presence of the hand held UV lamp. Surprisingly, the coated portion of the filter paper turns into bright cyan color fluorescence (Figure 7c), when illuminated under UV light. After that the filter paper was dried to evaporate the acid vapors which cause the compound coated filter paper to lose fluorescence (Figure 7c). Remarkably, the bright cyan fluorescence is regenerated when the filter paper comes in contact with any of the acid vapors (formic acid, acetic acid, trifluoreacetic acid, $\mathrm{HCl}, \mathrm{HNO}_{3}, \mathrm{H}_{2} \mathrm{SO}_{4}$ ). However, in the presence of $\mathrm{NH}_{3}$ vapor this fluorescence vanishes with a few seconds (figure 8). The fluorescence "on" and "off" behavior in the presence of acid and base vapors (Figure 8) indicates a fluorescence switch for the detection of acid and base in the gas phase and the process is recycled (Figure 9) for many times. Aggregation-induced fluorescence is responsible for the fluorescence "on" state and it is triggered in presence of acid vapors, whereas disaggregation of peptide containing NDI molecule occurs in the presence of base that leads to complete loss of fluorescence.

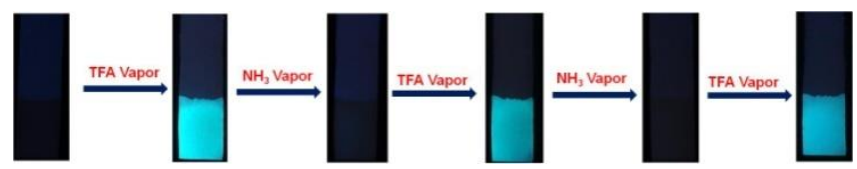

Figure 8. Fluorescence 'on/off' switching upon exposure to TFA and ammonia vapors of the paper strip device impregnated with NDIP.

Time-correlated single photon counting experiment (TCSPC) was carried out to get an information about differential nature of fluorescence life time and decay profile of the gelator molecule NDIP in the acid medium (Figure S10) and in the aggregated state (in phosphate buffer or in toluene). The excitation and emission monochromator were set at $340 \mathrm{~nm}$ and $480-500 \mathrm{~nm}$ respectively. The experiment was carried out in $\mathrm{HCOOH}$, as the compound NDIP gives the same fluorescence nature in different acid medium. It has been found that the average life time of NDIP in formic acid medium is $9.07 \mathrm{~ns}$ and this value is much higher than that of the aggregated state in phosphate buffer $(1.17 \mathrm{~ns})$ or in toluene $(1.01 \mathrm{~ns})$. So, it can be stated that excited state of NDIP in acid medium is more stable compared to that of its aggregated state (in phosphate buffer or in toluene medium). The sensitivity of NDIP towards volatile acids vapor was determined by change of fluorescence spectra upon the addition of NDIP to the different concentrated solutions of trifluoroacetic acid (TFA). In this study, eight sets of different concentrations of trifluoroacetic acid (starting from $0 \mathrm{ppm}$ to $200 \mathrm{ppm}$ ) were prepared by using milli-Q water. Then $10 \mu \mathrm{L}$ of the stock solution of NDIP (25 mM) in HFIP was added to the each set of TFA solution in such a way that final concentration of NDIP was 0.5 $\mathrm{mM}$. From the fluorescence spectra of NDIP (Figure 9b) it was observed that in absence of TFA ( $0 \mathrm{ppm})$ the peak appeared at $410 \mathrm{~nm}$. When the concentration of TFA was $4 \mathrm{ppm}$, the peak position at $410 \mathrm{~nm}$ was started to decrease and a new peak position centered at $483 \mathrm{~nm}$ started to appear. When the concentration of acid is more than $4 \mathrm{ppm}$ the peak position at $410 \mathrm{~nm}$ was fully diminished and the peak at $483 \mathrm{~nm}$ was started to increase (Figure 9b). So, it can be stated that the minimum detection limit is $4 \mathrm{ppm}$.
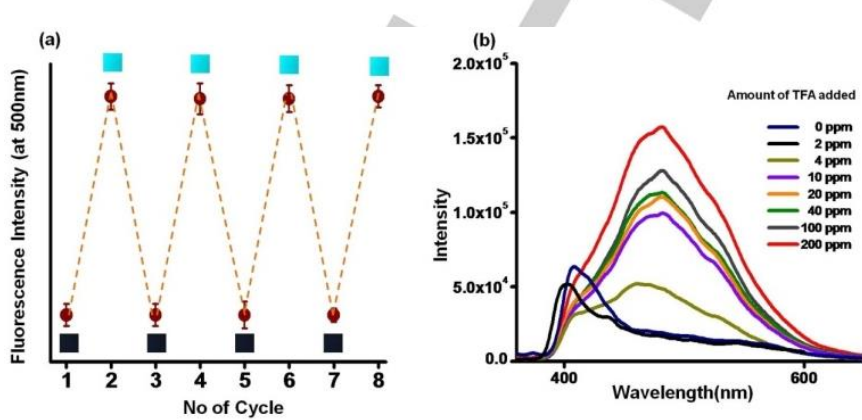

Figure 9. (a) Fluorescence on/off cycle of the compound NDIP in presence of acid and base vapors. (b) Fluorescence spectra of NDIP in milli-Q water in different amount (ppm) of trifluoroacetic acid (TFA).

To verify aggregation induced emission of the molecule in presence of acid, fluorescence measurements were performed at different concentrations starting from $5 \mathrm{mM}$ to $100 \mathrm{mM}$. It can be seen from the Figure $\mathrm{S} 11$ that the fluorescence intensity starts to increased when the concentration is $20 \mathrm{mM}$ and maximum intensity is obtained at $100 \mathrm{mM}$ NDIP. This shows that the molecule aggregates more with increasing concentration and, as a result, fluorescence develops.

\section{Conclusions}

This study demonstrates that a histidine-containing peptide conjugated naphthalenediimide based supramolecular species exhibits a 'turn on' fluorescence in the presence of hazardous acid vapors and 'turn off' fluorescence in ammonia vapor. An economically viable paper strip method has been used to detect fluorescence turn 'on' and 'off' and it is recyclable for several times. This indicates future promise for using naphthalenediimide- based molecular aggregates for sensing of environmentally hazardous volatile acid vapors in a cost effective manner.

\section{Experimental Section}

\section{Materials}

11-aminoundecanoic acid, 1,4,5,8 -Naphthalenetetracarboxylic dianhydride were purchased from Aldrich. L-histidine, HOBt (1hydroxybenzotriazole), DCC (N, N'-Dicyclohexylcarbodiimide), were purchased from SRL, India. Details synthetic procedure and spectroscopic analysis are given in the Supporting Information.

\section{Methods}


The compound NDIP was synthesized by conventional solution phase methods using racemisation free fragment condensation strategy. Couplings were mediated by DCC/HOBt. All compounds were purified by column chromatography using silica gel (100- 200 mesh size) as a stationary phase and chloroform and ethyl acetate as eluent. Finally, compounds were characterized by ${ }^{1} \mathrm{H}$ NMR, ${ }^{13} \mathrm{C}$ NMR and mass spectrometry.

\section{Gelation study , $\mathbf{T}_{\text {gel}}, \mathrm{MGC}$ (minimum gelation concentration)}

To test gelation, $6 \mathrm{mg}$ of compound was taken in a glass vial and it was dissolved in $1 \mathrm{ml}$ phosphate buffer $(50 \mathrm{mM})$ solution $\mathrm{pH} 7.46$ by heating on a hot plate. A transparent clear solution was found at first and that evolved into a self-supportive hydrogel (Figure 1a) after 25 minutes upon cooling down to room temperature. In a similar way, organogels were prepared by dissolving the compound in $5 \%$ methanol and benzene or toluene or o-xylene $(\mathrm{v} / \mathrm{v})$. Organogels were formed within a minute after obtaining a clear solution and just before attaining the room temperature. Organogels form much faster than the hydrogel at the same gelator concentration. The gel melting temperature $\left(T_{\text {gel }}\right)$ is higher for an organogel $\left(\mathrm{T}_{\text {gel }} 63^{\circ} \mathrm{C}\right.$ for benzene, $65^{\circ} \mathrm{C}$ for toluene and $72^{\circ} \mathrm{C}$ for xylene) than that of a hydrogel $\left(46^{\circ} \mathrm{C}\right)$ (Table S1). Moreover, the minimum gelation concentration (MGC [\%w/v]) is lower for organogel $(0.12 \mathrm{w} / \mathrm{v}$ for benzene and toluene, $0.11 \mathrm{w} / \mathrm{v}$ for xylene gel) than that of hydrogel $(0.14$ w/v) (Table S1).

\section{NMR experiments}

All NMR studies were carried out on a Bruker DPX400 MHz or Bruker DPX500 MHz spectrometer at $300 \mathrm{~K}$. Concentrations was in the range 5$10 \mathrm{mmol}$ in DMSO-d6.

\section{Mass spectrometry}

Mass spectra were recorded on a Q-TofmicroTM (Waters Corporation) mass spectrometer by a positive mode electrospray ionization process.

\section{MALDI-TOF MS}

MALDI-TOF MS analysis was performed using an Applied Biosystems MALDI-TOF Analyzer with dithranol as a matrix.

\section{Fourier Transform Infrared (FTIR) study}

All FT-IR spectra were recorded using the $\mathrm{KBr}$ pellet technique in a Nicolet 380 FT-IR spectrophotometer (Thermo Scientific).

\section{Powder X-ray diffraction study (XRD) study}

X-ray diffraction studies on the xerogels were carried out by placing the sample on a glass plate. Experiments were carried out using an X-ray diffractometer (Bruker AXS, Model D8 Advance). The instrument was operated at a $40 \mathrm{kV}$ voltage and $40 \mathrm{~mA}$ current using Ni-filtered CuKa radiation and the instrument was calibrated with a standard $\mathrm{Al}_{2} \mathrm{O}_{3}$ (corundum) sample before use. For scans over $2 \theta=1^{\circ}-5^{\circ}$, a scintillation counts detector was used with scan speed 2 s and step size $0.02^{\circ}$. In another $\operatorname{scan} 2 \theta=5^{\circ}-50^{\circ}$, a Lynx Eye super speed detector was used with scan speed 0.3 s and step size $0.02^{\circ}$.

Small-Angle X-ray Scattering (SAXS)
SAXS was performed on BM26B (DUBBLE) at the European Synchrotron Radiation Source, Grenoble, France. The sample-todetector distance was $2.095 \mathrm{~m}$ using a wavelength $\lambda=1.033 \AA$. A Dectris-PILATUS $1 \mathrm{M}$ detector with are solution of $981 \times 1043$ pixels and a pixel size of $172 \times 172 \mu \mathrm{m}$ was employed to record the 2DSAXS scattering patterns. Standard corrections for sample absorption and background subtraction were applied. The data were normalized to the intensity of the incident beam (in order to correct for primary beam intensity fluctuations) and were corrected for absorption, background scattering. The scattering pattern from rat tail collagen was used to calibrate the wave number $(q=4 \pi \sin \theta / \lambda)$ scale of the scattering curve. Samples were mounted in DSC pans modified with mica windows for transmission of the X-ray beam.

\section{Rheology}

The rheological experiments were carried out at $25^{\circ} \mathrm{C}$ using a Anton Paar Modular Compact Rheometer (Model-MCR 102). Parallel plate 7 was used as measuring system.

\section{Transmission electron microscopy (TEM)}

TEM images were recorded on a JEM 2010 electron microscope at an accelerating voltage of $200 \mathrm{KV}$. A drop of dilute solution of the gel-phase material were placed on carbon coated copper grids (300 mesh) and dried by slow evaporation. Each grid was then allowed to dry in a vacuum for two days and then images were taken.

\section{UV/Vis spectroscopy}

UV/Vis absorption spectra were recorded on a Hewlett-Packard (model 8453) UV/Vis spectrophotometer (Varian Carry 50.bio).

\section{PL spectroscopy}

Fluorescence studies of the gel were carried out in a Perkin Elmer LS55 Fluorescence Spectrometer instrument using the front face geometry. The sample was excited at $340 \mathrm{~nm}$ wavelength and emission scans were recorded from 350 to $750 \mathrm{~nm}$.

\section{Time-Correlated Single Photon Counting (TCSPC)}

TCSPC measurements were performed by Horiba Jobin Yvon IBH instrument having MCPPMT Hamamatsu R3809 detector.

\section{Preparation of thin film}

A thin film was prepared by drop casting of the organogel of the compound NDIP on a glass plate and it was dried in air oven.

\section{Preparation of paper based device}

A simple Whatman 1 filter paper with dimension $5 \mathrm{~cm} \times 0.5 \mathrm{~cm}$ was used. The filter paper was coated by the compound (dissolved in $10 \%$ methanol and chloroform) from the $1 \mathrm{~cm}$ bottom part and it was dried in an air oven for 10 minutes. 


\section{Acknowledgements}

K.G. and K.S.D. gratefully acknowledges CSIR, New Delhi and DST India for financial assistance respectively. K.B., N.N acknowledge IACS, India. The authors acknowledge Department of Science and Technology (DST-SERB) (DST project number: EMR/2016/005318), New Delhi for the grant. IWH acknowledges EPSRC, UK (EP/L020599/1).

\section{Conflict of interest}

The authors declare no conflict of interest.

Keywords: fluorescence sensing $\bullet$ hydrogel $\bullet$ naphthalene diimides $\cdot$ peptides $\bullet$ self-assembly

[1] a) S. Y. Lee, T. Yasuda, H. Komiyama, J. Lee, C. Adachi, Adv. Mater 2016, 28, 4019-4024; b) Y. Liu, C. Li, Z. Ren, S. Yan, M. R. Bryce, Nat Rev. Mater. 2018, 3, 1-20; c) S. Wang, X. Yan, Z. Cheng, H. Zhang, Y. Liu, Y. Wang, Angew. Chemie - Int. Ed. 2015, 54, 13068-13072.

[2] a)Z. Yuan, Y. Ma, T. Geßner, M. Li, L. Chen, M. Eustachi, R. T. Weitz, C. Li, K. Müllen, Org. Lett. 2016, 18, 456-459; b) R. Schmidt, J. H. Oh, Y.-S. Sun, M. Deppisch, A.-M. Krause, K. Radacki, H. Braunschweig, M. Könemann, P. Erk, Z. Bao, F. Würthner, J. Am. Chem. Soc. 2009, 131, 6215-6228; c) F. Würthner, M. Stolte, Chem. Commun. 2011, 47, 5109-5115.

[3] a) E. Zhou, J. Cong, Q. Wei, K. Tajima, C. Yang, K. Hashimoto, Angew. Chemie - Int. Ed. 2011, 50, 2799-2803; b) Z. Wu, C. Sun, S. Dong, X. F. Jiang, S. Wu, H. Wu, H. L. Yip, F. Huang, Y. Cao, J. Am. Chem. Soc 2016, 138, 2004-2013.

[4] a) E. R. Draper, L. J. Archibald, M. C. Nolan, R. Schweins, M. A. Zwijnenburg, S. Sproules, D. J. Adams, Chem. - A Eur. J. 2018, 24, 4006-4010; b) S. S. Babu, V. K. Praveen, A. Ajayaghosh, Chem. Rev. 2014, 114, 1973-2129; c) N. Dey, D. Biswakarma, A. Gulyani, S Bhattacharya, ACS Sustain. Chem. Eng. 2018, 6, 12807-12816; d) M. C. Nolan, J. J. Walsh, L. L. E. Mears, E. R. Draper, M. Wallace, M. Barrow, B. Dietrich, S. M. King, A. J. Cowan, D. J. Adams, J. Mater. Chem. A 2017, 5, 7555-7563; e) A. Sandeep, V. K. Praveen, K. K. Kartha, V. Karunakaran, A. Ajayaghosh, Chem. Sci. 2016, 7, 4460 4467 ; f) S. Roy, K. Basu, K. Gayen, S. Panigrahi, S. Mondal, D. Basak, A. Banerjee, J. Phys. Chem. C 2017, 121, 5428-5435.

[5] S. V. Bhosale, C. H. Jani, S. J. Langford, Chem. Soc. Rev. 2008, 37, 331-342.

[6] Y. Liu, L. Zhang, H. Lee, H. W. Wang, A. Santala, F. Liu, Y. Diao, A. L. Briseno, T. P. Russell, Adv. Energy Mater. 2015, 5, 2-9.

[7] a) S. V. Bhosale, S. V. Bhosale, M. B. Kalyankar, S. J. Langford, Org. Lett. 2009, 11, 5418-5421; b) W. Hughes, A. Rananaware, D. D. La, L. A. Jones, S. Bhargava, S. V. Bhosale, Sensors and Actuators B Chem. 2017, 244, 854-860.

[8] a) N. Singha, P. Gupta, B. Pramanik, S. Ahmed, A. Dasgupta, A. Ukil, D Das, Biomacromolecules 2017, 18, 3630-3641; b) F. Doria, M. Folini, V. Grande, G. Cimino-Reale, N. Zaffaroni, M. Freccero, Org. Biomol. Chem. 2015, 13, 570-576.

[9] a) S. Kuila, K. V. Rao, S. Garain, P. K. Samanta, S. Das, S. K. Pati, M. Eswaramoorthy, S. J. George, Angew. Chemie - Int. Ed. 2018, 57, 17115-17119; b) M. Kumar, S. J. George, Chem. - A Eur. J. 2011, $17,11102-11106$.
[10] a) N. Sakai, J. Mareda, E. Vauthey, S. Matile, Chem. Commun. 2010 46, 4225-4237; b) H. Kar, S. Ghosh, Chem. Commun. 2016, 52, 8818-8821; c) P. M. Alvey, B. L. Iverson, Org. Lett. 2012, 14, $2706-$ 2709; d) T. D. M. Bell, S. Yap, C. H. Jani, S. V. Bhosale, J. Hofkens, F. C. D. Schryver, S. J. Langford, K. P. Ghiggino, Chem. - An Asian J. 2009, 4, 1542-1550.

[11] a) S. Basak, J. Nanda, A. Banerjee, Chem. Commun. 2013, 49, 68916893; b) S. Basak, N. Nandi, A. Baral, A. Banerjee, Chem. Commun 2015, 51, 780-783.c) N. Nandi, K. Gayen, A. Banerjee, Soft Matter 2019, 15, 3018-3026. d) b. S. Basak, N. Nandi, S. Paul, A. Banerjee ACS Omega 2018, 3, 2174-2182.

[12] a) P. Chakraborty, T. Guterman, N. Adadi, M. Yadid, T. Brosh, L. AdlerAbramovich, T. Dvir, E. Gazit, ACS Nano 2019, 13, 163-175; b) J. A. Foster, R. M. Edkins, G. J. Cameron, N. Colgin, K. Fucke, S. Ridgeway, A. G. Crawford, T. B. Marder, A. Beeby, S. L. Cobb, J. W. Steed, Chem. - A Eur.J. 2014, 20, 279-291. C) V. Castelletto, I. W. Hamley, J. Seitsonen, J. Ruokolainen, G. Harris, K. Bellmann-Sickert, A. G. BeckSickinger, Biomacromolecules 2018, 19, 4320-4332; d) W. Edwards, D. K. Smith, J. Am. Chem. Soc. 2013, 135, 5911-5920; e) M. C. Branco, J. P. Schneider, P. J. Knerr, R. Nagarkar, D. J. Pochan, J. Mater. Chem. 2012, 22, 1352-1357; f) M. Maity, V. S. Sajisha, U. Maitra, RSC Adv 2015, 5, 90712-90719; g) K. Nagy-Smith, P. J. Beltramo, E. Moore, R Tycko, E. M. Furst, J. P. Schneider, ACS Cent. Sci. 2017, 3, 586-597. h) N. Singh, M. Kumar, J. F. Miravet, R. V. Ulijn, B. Escuder, Chem. - A Eur. J. 2017, 23, 981-993; i) J. Rubio-Magnieto, M. Tena-Solsona, B. Escuder, M. Surin, RSC Adv. 2017, 7, 9562-9566; j) D. B. Amabilino, D. K. Smith, J. W. Steed, Chem. Soc. Rev. 2017, 46, 2404-2420; k) M. Amit, S. Yuran, E. Gazit, M. Reches, N. Ashkenasy, Adv. Mater. 2018, 30, 1-13.

[13] a) N. Chuard, K. Fujisawa, P. Morelli, J. Saarbach, N. Winssinger, P. Metrangolo, G. Resnati, N. Sakai, S. Matile, J. Am. Chem. Soc. 2016 138, 11264-11271; b) S. K. M. Nalluri, C. Berdugo, N. Javid, P. W. J. M. Frederix, R. V. Ulijn, Angew. Chemie - Int. Ed. 2014, 53, 5882-5887; c) S. Basak, S.Bhattacharya, A. Datta, A. Banerjee, Chem. - A Eur. J. 2014, 20, 5721-5726; d) S. Basak, N. Nandi, K. Bhattacharyya, A Datta, A. Banerjee, Phys. Chem. Chem. Phys. 2015, 17, 30398-30403; e) M. B. Avinash, K. Swathi, K. S. Narayan, T. Govindaraju, ACS Appl. Mater. Interfaces 2016, 8, 8678-8685; f) L. H. Hsu, S. M. Hsu, F. Y. Wu, Y. H. Liu, S. R. Nelli, M. Y. Yeh, H. C. Lin, RSC Adv. 2015, 5, 2041020413; g) F. K. Zhan, S. M. Hsu, H. Cheng, H. C. Lin, RSC Adv. 2015, 5, 48961-48964; h) D H. Shao, T. Nguyen, N. C. Romano, D. A. Modarelli, J. R. Parquette, J. Am. Chem. Soc. 2009, 131, 16374 16376; i) S. P. Goskulwad, M. Al Kobaisi, D. D. La, R. S. Bhosale, M. Ratanlal, S. V. Bhosale, S. V. Bhosale, Chem. - An Asian J. 2018, 13 , 3947-3953; j) N. Ponnuswamy, G. D. Pantoş, M. M. J. Smulders, J. K. M. Sanders, J. Am. Chem. Soc. 2012, 134, 566-573; k) N. Nandi, S. Basak, S. Kirkham, I. W. Hamley, A. Banerjee, Langmuir 2016, 32, 13226-13233.

[14] J. Geltmeyer, G. Vancoillie, I. Steyaert, B. Breyne, G. Cousins, K. Lava, R. Hoogenboom, K. De Buysser, K. De Clerck, Adv. Funct. Mater. 2016, 26, 5987-5996.

[15] M. E. Genovese, A. Athanassiou, D. Fragouli, J. Mater. Chem. A 2015 3, 22441-22447.

[16] a) X. Yang, Y. Liu, J. Li, Q. Wang, M. Yang, C. Li, New J. Chem. 2018 42, 17524-17532; b) P. Xue, J. Ding, Y. Shen, H. Gao, J. Zhao, J. Sun, R. Lu, J. Mater. Chem. C 2017, 5, 11532-11541; c) Y. Zhan, P. Yang, G. Li, Y. Zhang, Y. Bao, New J. Chem. 2016, 41, 263-270; d) P. Xue, B. Yao, Y. Shen, H. Gao, J. Mater. Chem. C 2017, 5, 11496-11503.

[17] (a)Q. Li, M. Peng, H. Li, C. Zhong, L. Zhang, X. Cheng, X. Peng, Q. Wang, J. Qin, Z. Li, Org. Lett. 2012, 14, 16160-16161; b) M. Pandeeswar, S. P. Senanayak, T. Govindaraju, ACS Appl. Mater. Interfaces 2016, 8, 30362-30371.

[18] A. Kalita, S. Hussain, A. H. Malik, U. Barman, N. Goswami, P. K. Iyer, ACS Appl. Mater. Interfaces 2016, 8, 25326-25336. 
[19] J. Fan, X. Chang, M. He, C. Shang, G. Wang, S. Yin, H. Peng, ACS Appl. Mater. Interfaces 2016, 8, 18584-18592

[20] a) F. Doria, A. Oppi, F. Manoli, S. Botti, N. Kandoth, V. Grande, I. Manet, M. Freccero, Chem. Commun. 2015, 51, 9105-9108; b) G. W. Collie, R. Promontorio, S. M. Hampel, M. Micco, S. Neidle, G. N Parkinson, J. Am. Chem.Soc. 2012, 134, 2723-2731.

[21] a) G. Palui , F.-X. Simon, M. Schmutz , P. J. Mesini , A. Banerjee, Tetrahedron 2008, 64, 175-185. b) A. Motulskya, M. Lafleurb, A.-C. Couffin-Hoaraua, D. Hoarauc, F. Bouryd, J.-P. Benoitd, J.-C. Leroux Biomaterials 2005, 26, 6242-6253. c) A. Banerjee, G. Palui, A. Banerjee, Soft Matter 2008, 4, 1430-1437.

[22] a) I.W.Hamley, Angew. Chem. Int. Ed. 2014, 53, 6866-6881. b) I. W. Hamley, A. Dehsorkhi, V. Castelletto,S. Furzeland, D. Atkins, J. Seitsonen , J. Ruokolainen, Soft Matter 2013, 9, 9290-9293.

[23] J. Naskar, G. Palui, A. Banerjee, J. Phys. Chem. B 2009, 113, 1178711792 


\section{FULL PAPER}

A peptide appended naphthalenediimide was found to form fluorescent gels both in organic and aqueous media. The fluorescence was found to be quenched in the respective xerogel states. However, it reappears upon exposure to the acid vapour. A paper strip based fluorescence switch has been constructed with this gelator molecule that exhibits 'turn on' and 'turn off' fluorescence behaviour in presence of acid and base vapors respectively with recyclability for at least seven times.

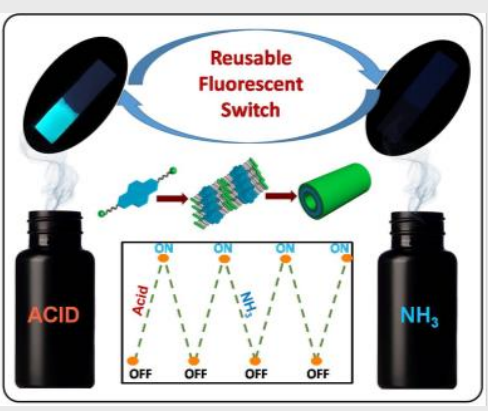

Kousik Gayen, [a] Kingshuk Basu, [a] Nibedita Nandi, ${ }^{[a]}$ Krishna Sundar Das, ${ }^{[b]}$ Daniel Hermida-Merino, ${ }^{[c]}$ Ian W. Hamley ${ }^{[d]}$ and Arindam Banerjee ${ }^{\star[a]}$

\section{Page No. - Page No.}

A Self-Assembled Peptide-Appended Naphthalene Diimide: A Fluorescent Switch for Sensing Volatile Acid and Basic Vapors 